REVISION

\title{
EDUCACION INTERCULTURAL EN AMERICA LATINA: DISTINTAS CONCEPCIONES Y TENSIONES ACTUALES ${ }^{1}$
}

\author{
INTERCULTURAL EDUCATION IN LATIN AMERICA: \\ DIFFERENT CONCEPTIONS AND CURRENT TENSIONS \\ Educação intercultural na América Latina: diferentes concepções e tensões atuais \\ Vera Maria Ferrão Candau \\ Profesora Titular \\ Departamento de Educación. Pontifícia Universidad Católica de Rio de Janeiro \\ PUC-Río. Rua Marquês de São Vicente, 225 \\ Gávea - Rio de Janeiro, BRASIL \\ vmfc@puc-rio.br, tel. 55-21-22874957
}

\begin{abstract}
RESUMEN
La interculturalidad ha venido adquiriendo especial relevancia en América Latina, sobre todo a partir de los años noventa. El presente trabajo se ubica dentro de este contexto y forma parte de la investigación que venimos desarrollando desde 2006. Dar a conocer y discutir las tensiones presentes en el desarrollo de la educación intercultural en el continente constituye el foco del presente trabajo, el cual se estructura de la siguiente manera: en un primer momento presentaremos una breve síntesis de la evolución histórica de la educación intercultural en el continente para, en un segundo momento, focalizar y discutir las principales tensiones presentes hoy en relación con la educación intercultural. Para finalizar, destacaremos algunos desafíos que consideramos centrales para que la educación intercultural fortalezca los procesos democráticos en el continente.
\end{abstract}

Palabras clave: educación intercultural, América Latina, interculturalidad crítica.

\begin{abstract}
The interculturality is becoming more relevant in Latin America, especially since the 1990's. The present paper fits into this context and is part of the research we are developing since 2006. The main focus of this article is to present and discuss the tensions experienced in the development of intercultural education in the continent. This paper begins with a small synthesis of the historical evolution of the intercultural education and its main agents. Secondly, it focuses on and discusses the main current tensions related to the intercultural education. Finally, we will outline some challenges which we consider fundamental so that the intercultural education may contribute to strengthening of the democratic processes in the continent.
\end{abstract}

Key-words: intercultural education, Latin America, critical interculturality.

\section{RESUMO}

A interculturalidade tem adquirido especial relevância na América Latina, principalmente a partir dos anos 1990. O presente trabalho se situa nesse contexto e integra uma pesquisa que estamos desenvolvendo desde 2006. Seu foco principal constitui em apresentar e discutir as tensões que vêm atravessando o desenvolvimento da educação intercultural no continente nos últimos anos. $\mathrm{O}$ trabalho, num primeiro momento, apresenta uma breve síntese da evolução histórica da educação intercultural e seus principais atores para, em seguida, focalizar-se e discutir

1 Trabajo realizado con el financiamiento del Consejo Nacional de Investigaciones (CNPq), órgano del Ministerio de Ciencia y Tecnología del gobierno brasileño. 
as principais tensões presentes hoje na América Latina em relação à educação intercultural. Termina assinalando alguns desafios para que a educação intercultural possa contribuir para fortalecer os processos democráticos no continente.

Palavras-chave: educação intercultural, América Latina, interculturalidade crítica.

\section{INTRODUCCION}

La interculturalidad ha venido adquiriendo especial relevancia en América Latina, sobre todo a partir de los años noventa. En diversos países del continente ha sido causa de una intensa producción bibliográfica y de fuertes discusiones. Sus diferentes dimensiones -política, ética, social, jurídica, epistemológica y educacional- además de haber sido analizadas han sido objeto de debates de agentes de la sociedad civil, así como ocasión para el aumento de las investigaciones, entre otras iniciativas -cursos, seminarios, congresos, etc.-, en el ámbito académico. La interculturalidad también ha servido de estímulo para la elaboración de políticas públicas que, o se centran en ella, o la contemplan.

El presente trabajo se ubica dentro de este contexto y forma parte de la investigación que venimos desarrollando desde 2006, mediante el grupo de investigación que coordinamos -Grupo de Estudios sobre Cotidianeidad, Educación y Cultura(s) (GECEC)-, sobre la génesis histórica y la problemática actual de las relaciones entre interculturalidad y educación en el continente. Con este fin, realizamos una amplia revisión bibliográfica sobre la educación intercultural en América Latina y, de manera particular en las últimas décadas, hemos efectuado entrevistas con especialistas y militantes de movimientos sociales de diferentes procedencias, además de analizar la incorporación de la interculturalidad en las políticas educacionales de varios países. Participamos también de muchos seminarios y encuentros sobre dicha temática. El análisis de los datos recolectados permitió no sólo identificar las tensiones que traspasaban la discusión, sino también las propuestas surgidas sobre educación intercultural en el continente. Análisis que, valga la aclaración, consideramos de especial relevancia.

Dar a conocer y discutir esas tensiones constituye el foco del presente texto, el cual se estructura de la siguiente manera: en un primer momento presentaremos una breve síntesis de la evolución histórica de la educación intercultural en el continente y de sus principales agentes para, en un segundo momento -la parte central de este trabajo-, focalizar y discutir las principales tensiones presentes hoy en América Latina en relación con la educación intercultural. Para finalizar, terminaremos destacando algunos desafíos que consideramos centrales para que la educación intercultural fortalezca los procesos democráticos en el continente.

\section{EDUCACION INTERCULTURAL EN AMERICA LATINA: UN CAMINO COMPLEJO Y ORIGINAL}

Toda la producción bibliográfica analizada, así como los testimonios de los/as entrevistados/as de los diferentes países, fueron unánimes en afirmar que el término "interculturalidad" surge en América Latina dentro del contexto educacional y, más específicamente, vinculado a la educación escolar indígena. Según López-Hurtado Quiroz 
(2007: 15), Mosonyi y González, dos lingüistas antropólogos venezolanos, son los primeros en definir el concepto de interculturalidad, en la primera mitad de los años 70, para aplicarlo al tema educativo y utilizarlo para describir sus experiencias con los indígenas arhuacos, de la región de Río Negro, en Venezuela.

A pesar de ser conscientes de la diversidad de trayectorias que ha sufrido la educación escolar indígena en los diferentes países y contextos, es posible afirmar que su desarrollo en el continente ha seguido cuatro etapas fundamentales.

La primera, que va del período colonial hasta las primeras décadas del siglo XX, se puede caracterizar por una violencia etnocéntrica explícita, que intenta imponer la cultura hegemónica sobre las poblaciones indígenas. Eliminar al "otro" fue la tónica del período colonial. Sin embargo, a partir de las primeras décadas del siglo XX, esa eliminación adquiere otra forma: la "asimilación", base para la construcción de la homogeneidad requerida por los Estados nacionales modernos. En esta segunda etapa surgieron las primeras escuelas bilingües dirigidas a los pueblos indígenas. Por primera vez, otras lenguas se incorporaban al espacio escolar, además de la oficial. Pero, salvo raras excepciones, estas escuelas veían el bilingüismo simplemente como una etapa de transición necesaria: un modo de alfabetizar y "civilizar" más fácilmente a pueblos enteros. Fue esta concepción de bilingüismo la que influyó en las políticas educativas dirigidas a las comunidades indígenas en toda América Latina hasta la década del 70, época en que se da comienzo a una tercera etapa de desarrollo de la educación escolar indígena, a partir de las experiencias alternativas protagonizadas por líderes comunitarios, como resultado de una acción conjunta con universidades y sectores progresistas de la iglesia católica. En este nuevo período se produjeron materiales didácticos alternativos y programas de educación bilingüe $\mathrm{y}$, pese a que aún se está en la búsqueda de que los mismos contribuyan a una mayor "integración" entre los grupos y las sociedades nacionales, dichos materiales reconocían el derecho de esos pueblos de fortalecer y mantener su propia cultura. El bilingüismo deja de ser visto como mero instrumento civilizatorio, para ser considerado de importancia fundamental para la continuidad de los propios grupos minoritarios. Dentro de esa nueva conformación, el bilingüismo pasa a formar parte de un discurso más amplio, en el que la perspectiva intercultural presiona el modelo escolar clásico y en el que se incluyen, no solamente diferentes lenguas, sino, y sobre todo, diferentes culturas. A partir de los años 80, luchas indígenas, antes aisladas, protagonizadas por cada etnia en particular, empezaron a unirse bajo una identidad común: la "indígena", y comenzaron a adquirir en el exterior, no sólo mayor reconocimiento, sino también mayor espacio. López y Sichra (2004) recuerdan que, tanto en los países con una población mayoritariamente indígena, como es el caso de Bolivia (con población indígena de aproximadamente un 65\%), como en aquellos países en que la población indígena es minoritaria, como es el caso de Brasil (con un 0,3\% de indígenas), ha surgido cada vez con más fuerza una exigencia común por escuelas coordinadas y dirigidas por profesores indígenas. La experiencia de escuelas interculturales indígenas que se han desarrollado en el continente incluyó una nueva dimensión con relación a la idea de cultura en el espacio escolar. Las diferentes lenguas fueron el primer paso para que se propusiera un diálogo entre las diferentes culturas.

Además de la educación escolar indígena, otros grupos contribuyeron para que creciera la discusión sobre las relaciones entre educación e interculturalismo. Entre ellos, podemos mencionar a los movimientos negros latinoamericanos que, en general, son 
ignorados por la bibliografía que trata de educación intercultural en el continente. Sin embargo, a nuestro ver esos grupos contribuyen de modo significativo para la ampliación del concepto de educación intercultural.

A pesar de que la realidad de los grupos y movimientos negros sea muy heterogénea y diferenciada en la región, es posible afirmar que, en general, a estos grupos se los redujo a una posición de no ciudadanía hasta la mitad del siglo pasado. Vale recordar que el régimen de esclavitud subsistió en algunos países, como en el Brasil, hasta fines del siglo XIX. Sin embargo, en diferentes naciones, diversos grupos afrodescendientes llevaron a cabo luchas por condiciones de vida más dignas y por el combate a la discriminación y al racismo. Estos grupos se han caracterizado por la resistencia y por sus luchas contra el racismo en sus diferentes manifestaciones, así como por la afirmación de los derechos y de la plenitud de la ciudadanía, lo que supone reconocimiento de sus identidades culturales. Algunas de las contribuciones de la educación intercultural que podemos citar son: la denuncia de las diferentes manifestaciones de discriminación racial presentes en las sociedades latinoamericanas; el combate a la ideología del mestizaje y a la "democracia racial", que configura el imaginario sobre las relaciones sociales y raciales que se ha mantenido entre los diferentes grupos presentes en las sociedades latinoamericanas, y que presenta estas relaciones como caracterizadas por la cordialidad. De esta manera, el conflicto es eliminado y se continúan perpetuando estereotipos y prejuicios. Los movimientos negros organizados han promovido lecturas alternativas acerca del proceso histórico vivido y acerca del papel de los negros en la formación de varios países latinoamericanos. También presentaron demandas a los Estados por reparaciones e indemnizaciones para los afrodescendientes, por los daños sufridos bajo el régimen de esclavitud, así como por las políticas explícitas o tácitas de emblanquecimiento de la población. En lo que se refiere a la educación, se han incluido en varios países políticas cuyo objetivo era el ingreso, la permanencia y el éxito en la educación escolar; la valoración de las identidades culturales negras; la incorporación, tanto en los currículos escolares como en los materiales pedagógicos, de componentes propios de las culturas negras, de los procesos históricos de resistencia vividos por los grupos negros, así como de las contribuciones de estos grupos para la construcción histórica de los diferentes países. Otro elemento importante que se ha venido incorporando en algunos países son las políticas de acción afirmativa dirigidas a los afrodescendientes, en diferentes ámbitos de la sociedad, del mercado de trabajo a la enseñanza superior. Son propuestas como éstas las que ponen en discusión el discurso y las prácticas eurocéntricas, homogeneizadoras y monoculturales de los procesos sociales y educativos y ubican en el escenario político asuntos relacionados con la construcción de relaciones étnico-raciales en los contextos latinoamericanos.

Otra contribución importante para el carácter particular del desarrollo de la educación intercultural en el continente tiene que ver con las experiencias de educación popular realizadas a lo largo de toda América Latina, particularmente, a partir de los años 60. Al igual que las anteriores, esta contribución también se da en un universo heterogéneo y cuenta con diferentes repercusiones en los diversos contextos. Si bien estas experiencias privilegiaron los ámbitos de educación no formal -principalmente a partir de fines de la década del 80 y comienzos de los años 90-, el hecho es que dejaron una impronta en las propuestas de renovación de diversos sistemas escolares. Su principal contribución, desde la perspectiva trabajada en el presente texto, ha consistido en afirmar la intrínseca articulación existente entre los procesos educativos y los contextos socioculturales en los 
que aquellos se desarrollan. Con esto, los universos culturales de los actores implicados acabaron siendo incorporados en el centro de las acciones pedagógicas. Desde esta perspectiva, la contribución de Paulo Freire ha sido fundamental.

Un cuarto movimiento que ha intensificado la importancia del tema multicultural en el continente data de las décadas del 80 y del 90, época en que innúmerables países latinoamericanos reconocieron en sus Constituciones el carácter multiétnico, pluricultural y multilingüe de sus sociedades. Como consecuencia, las políticas públicas en el área educativa se han visto en la necesidad de contemplar las diferencias culturales y las diversas reformas en educación han incorporado la perspectiva intercultural, ya sea instituyéndola en uno de los ejes de articulación de los currículos escolares, ya sea introduciendo en los temas transversales cuestiones relativas a las diferencias culturales. Sin embargo, si esta ampliación del concepto y de su impacto sobre las políticas públicas puede ser vista como un progreso significativo, no por ello deja de estar traspasada por fuertes ambigüedades, ya que dicha incorporación se da en el contexto de gobiernos comprometidos con la implementación de políticas de carácter neoliberal, que asumen la lógica de la globalización hegemónica y la agenda de los principales organismos internacionales.

Teniendo en cuenta el proceso que ha vivido la educación intercultural en el continente, de manera global, podemos asumir, junto con López-Hurtado Quiroz (2007: 21-22), la siguiente síntesis que da cuenta de su incorporación en la agenda latinoamericana:

En estos treinta años, desde que fue acuñado y aceptado en la región, el término trascendió el ámbito de los programas y de los proyectos que se referían a los indígenas y, hoy, un número importante de países, desde México a Tierra del Fuego, ven en él una posibilidad de transformar tanto a la sociedad como un todo como a los sistemas educativos nacionales, en el sentido de crear una articulación más democrática entre las diferentes sociedades y pueblos que integran un determinado país. Desde este punto de vista, hoy la interculturalidad también supone apertura frente a las diferencias étnicas, culturales y lingüísticas; a la aceptación positiva de la diversidad; al respeto mutuo; a la búsqueda de consenso y, paralelamente, al reconocimiento y aceptación del disenso y, actualmente, a la construcción de nuevos modos de relación social y de más democracia.

\section{EDUCACION INTERCULTURAL EN AMERICA LATINA: TENSIONES ACTUALES}

Una lectura crítica sobre el proceso de estas últimas décadas nos permite afirmar que la perspectiva de la interculturalidad admite diferentes búsquedas y preocupaciones, tanto desde el punto de vista de la reflexión teórica como desde el punto de vista de las iniciativas concretas, especialmente en el ámbito de la educación. Se trata de una problemática compleja que se halla traspasada por diferentes tensiones. De estas, intentaremos analizar aquellas que consideramos fundamentales.

\subsection{INTERCULTURALIDAD FUNCIONAL VS. INTERCULTURALIDAD CRITICA}

La primera tensión que quisiéramos destacar tiene que ver con las relaciones entre la interculturalidad y la dinámica de la sociedad, en general. Muchas han sido las propuestas para identificar diferentes concepciones de interculturalidad, explícita o implícitamente, en los diversos discursos y/o prácticas. Resaltamos la postura de Fidel Tubino (2005), autor 
peruano que se viene dedicando a discutir la temática que nos ocupa, por considerarla particularmente esclarecedora. En su texto "La interculturalidad crítica como proyecto ético-político", distingue dos perspectivas fundamentales: la interculturalidad funcional y la interculturalidad crítica.

El autor parte de la afirmación de que la creciente incorporación de la interculturalidad en el discurso oficial de los estados y organismos internacionales se ha apoyado, en la mayoría de los países, en una postura de no cuestionamiento del modelo sociopolítico vigente, la cual, además, se encuentra marcada por la lógica neoliberal. Es decir, como afirma el autor, "no pone en duda las reglas del juego" (Tubino, 2005:3). En este sentido, la interculturalidad se asume como una estrategia que favorece la cohesión social, en tanto y en cuanto asimila a los grupos socioculturales subalternizados a la cultura hegemónica. Se trata de "promover el diálogo y la tolerancia sin afectar las causas de la asimetría social y cultural actualmente vigentes" (Tubino, 2005:5). Las relaciones de poder entre los diferentes grupos socioculturales no son puestos en duda. De esta manera, el interculturalismo funcional tiende a disminuir las áreas de tensión y conflicto entre los diversos grupos y movimientos sociales, cuyo foco de atención son las cuestiones socioidentitarias, evitando que la estructura y las relaciones de poder vigentes sean afectadas.

Sin embargo, poner en duda estas relaciones es exactamente el foco de la perspectiva de la interculturalidad crítica. Se trata de cuestionar las diferencias y desigualdades construidas a lo largo de la historia entre diferentes grupos socioculturales, étnico-raciales, de género, de orientación sexual, entre otros. Se parte de la afirmación de que la interculturalidad apunta hacia la construcción de sociedades que asuman las diferencias como constitutivas de la democracia y sean capaces de construir relaciones nuevas, verdaderamente igualitarias entre los diferentes grupos socioculturales, lo que supone empoderar a aquellos que, históricamente, fueron considerados inferiores.

Según Tubino (2005: 5):

La asimetría social y la discriminación cultural no permiten un diálogo intercultural auténtico. Por eso no hay que comenzar por el diálogo, sino por cuestionarnos cuáles son las condiciones existentes de diálogo. $\mathrm{O}$, para ser más precisos, es necesario exigir que el diálogo entre las culturas sea, en primer lugar, un diálogo sobre los factores económicos, políticos, militares, etc, que condicionan actualmente el intercambio franco entre las culturas de la humanidad. Esta exigencia se hace hoy imprescindible para que no caigamos en la ideología de un diálogo descontextualizado, que se limita a favorecer los intereses creados de la civilización dominante, y que no toma en consideración la asimetría de poder que hoy reina en el mundo. Para que el diálogo sea real, es necesario hacer visibles las causas del no diálogo, lo que pasa, necesariamente, por un discurso de crítica social.

La interculturalidad crítica pretende ser una propuesta ética y política con el objetivo de construir sociedades democráticas que articulen la igualdad y el reconocimiento de las diferentes culturas, y de proponer alternativas al carácter monocultural occidentalizante, que domina en la mayoría de los países del continente.

Estas dos perspectivas se cruzan, se chocan y algunas veces se articulan contradictoriamente en las diversas búsquedas, experiencias y propuestas que se llevan a cabo en el continente. Esta es la principal tensión que se halla presente en el debate sobre las relaciones entre interculturalidad y educación, hoy en América Latina, y que traspasa a todas las demás. 


\subsection{INTERCULTURALIDAD PARA ALGUNOS/AS VS. INTERCULTURALIDAD PARA TODOS/AS}

En cuanto a la segunda tensión, la Interculturalidad para algunos/as vs. la Interculturalidad para todos/as, la misma está relacionada con el origen de esta preocupación en el continente: la educación escolar indígena. Dentro de esta perspectiva, la educación intercultural se dirigía exclusivamente a los grupos subalternizados, generalmente étnicoraciales y sobre todo a los indígenas y, menos frecuentemente, a los afroamericanos. Son estos grupos los "otros", los "diferentes" y los que, desde la óptica de la interculturalidad funcional, deben ser integrados a la sociedad nacional.

Sin embargo, y particularmente a partir de la década del 90, se ha venido afianzando la postura de que la interculturalidad debe ser trabajada por todos los agentes sociales, si realmente queremos que se vuelva una característica de la sociedad como un todo, en su proceso de construcción democrática. La perspectiva de la interculturalidad crítica acentúa este aspecto e intenta trabajarlo a partir de sus presupuestos.

A pesar de que en los últimos años esta preocupación ha cobrado mayor firmeza, tanto en la bibliografía que hemos analizado como en las entrevistas que realizamos en diferentes países, con frecuencia hemos oído sobre la resistencia social existente en relación con este tema, así como sobre la dificultad de asumir la perspectiva intercultural en la educación de todos/as los/as ciudadanos/as.

Esta problemática fue abordada en las entrevistas que realizamos con especialistas, sobre todo en Perú y en Bolivia (Russo y Drelich, 2009; Sacavino y Pedreira, 2009). Las causas apuntadas como originarias de la dificultad de asumir la educación intercultural para todos/as fueron, principalmente, las siguientes: la existencia de un fuerte racismo en la sociedad, muchas veces velado y encubierto por un discurso que defiende el mestizaje, que niega las diferencias culturales y que ve inadecuado que se introduzcan aspectos relativos a diferentes grupos socioculturales en el currículo escolar, con el pretexto de que fragiliza la cultura común y la cohesión social. Según varios entrevistados, el pensamiento colonial aún es dominante en la sociedad, lo que lleva a que se enfatice y se considere superior la lógica europeizante y de influencia norteamericana, y a que se le dé poco valor a las culturas originarias y/o afroamericanas. Otra causa apuntada para la presencia precaria de la perspectiva intercultural en las escuelas fue la ausencia de esta temática en los centros de formación de profesores/as.

\subsection{EDUCACION INTERCULTURAL VS. INTERCULTURALIDAD COMO PROYECTO POLITICO}

Esta tensión puede considerarse como un desdoblamiento de los aspectos mencionados en el punto anterior. La afirmación unánime de que la preocupación con la interculturalidad nace en América Latina en el contexto de la educación y, concretamente, en el ámbito de la educación escolar indígena, trajo como consecuencia la visión de que es este ámbito, entonces, su locus específico. Esta visión pretende reducir las contribuciones que la Interculturalidad puede ofrecerle al campo educativo, las que, como mucho, podrían extenderse a la educación de otros grupos existentes en la sociedad. Se trata de una cuestión exclusivamente educativa y restricta a algunos grupos, a los "otros", considerados diferentes, para favorecer su inserción en el sistema escolar vigente. La educación intercultural se limita a introducir algunos contenidos relativos a diferentes culturas, sin que esto afecte al currículo como un todo, ni afecte la predominancia de la considerada 
“cultura común" y de los conocimientos y valores considerados "universales". Una vez más, se asume la perspectiva de la interculturalidad funcional.

Sin embargo, otras visiones inspiradas en la interculturalidad crítica defienden su incorporación en diferentes ámbitos de la sociedad: el jurídico, el de la salud, el del medio ambiente, el de la economía, el de la producción cultural y el de la política. Por otra parte, en lo que se refiere al ámbito educativo, no reducen la interculturalidad a lo que podríamos llamar un enfoque aditivo, sino que pretenden promover una transformación curricular que afecte todos sus componentes y que cuestione la construcción de la llamada "cultura común" y de los conocimientos y valores considerados "universales". Preguntas tales como: ¿En que consiste y cómo se construye la "cultura común”? ¿Cuáles son los agentes sociales que intervienen en dicha construcción? ¿Cómo se define todo aquello que conforma lo que consideramos "universal"?, se hacen fuertemente presentes en esta perspectiva dirigida a repensar la epistemología que preside las formulaciones curriculares vigentes en nuestras sociedades, con el objetivo de impulsar el diálogo entre diferentes cosmovisiones y saberes inherentes a los diversos grupos socioculturales, y dirigida, además, a proponer que la educación intercultural se desarrolle conjuntamente con la implementación de prácticas interculturales en diferentes ámbitos sociales.

En este sentido, la interculturalidad se ubica no solamente en un horizonte político de construcción de estados pluriculturales y multilingües, sino que, para algunos, también se ubica en el horizonte de construcción de estados plurinacionales. Este es un tema controvertido que atraviesa las luchas políticas que se han impulsado para la elaboración de nuevas constituciones, principalmente en el Ecuador y en Bolivia, y que le presenta varios cuestionamientos a las teorías políticas de origen europea a las que estamos acostumbrados a tener como referencia. Es posible afirmar que se está gestando en el continente una nueva concepción de Estado, de democracia y de ciudadanía, en que la interculturalidad crítica constituye el elemento central.

\subsection{INTERCULTURALIDAD VS. INTRACULTURALIDAD}

En los últimos años, especialmente en algunos países, se ha venido discutiendo la noción de intraculturalidad. En las entrevistas que realizamos con especialistas bolivianos (Sacavino y Pedreira 2009), los mismos se referían a este tema de la siguiente manera: se trata de: "un proceso de mirar la cultura y valorar lo que tenemos adentro: cultura, lengua, costumbres, tradiciones, leyes, etc.", considerando que "[...] para poder reconocernos, para poder saber quiénes somos, necesitamos aprender lo que nos han dado los abuelos. Y la intraculturalidad no resulta ser otra cosa que el aprendizaje al interior del grupo cultural donde yo me desarrollo". Los entrevistados atribuyeron el origen reciente del término a Félix Pacci, primer indígena en convertirse en Ministro de Educación en Bolivia, tras haber alertado sobre el riesgo de que la interculturalidad asumiera un carácter homogeneizador, frente al poder de la cultura dominante occidental.

El papel de la intraculturalidad y las relaciones entre la inter y la intraculturalidad suscitan diferentes posturas. Para algunos, la intraculturalidad es el fortalecimiento de la identidad propia de cada grupo y se la considera condición necesaria para el desarrollo de procesos interculturales. No siendo así, puede favorecer el debilitamiento y la dilución de la propia identidad. Sin embargo, hay quienes resaltan que el énfasis en la intraculturalidad puede favorecer el etnocentrismo e inhibir la apertura hacia los otros. 
También se habla sobre la necesidad de tener una visión dinámica de la propia cultura, de modo que se evite una perspectiva esencialista, ahistórica, que fosiliza las tradiciones culturales. La relación entre intra e interculturalidad es vista por algunos, no como momentos sucesivos, sino como un proceso interrelacionado. La interculturalidad debe contribuir a la toma de conciencia de la propia identidad cultural, la cual, en general, halla dinamismo en el contraste con el "Otro", con el "diferente". Consiste, por lo tanto, en procesos imbricados y entrelazados. Es importante, también, estar alerta a que, para que la interculturalidad sea auténtica, debe promover un diálogo con bases igualitarias. $\mathrm{Y}$ esto no se da por presupuesto, sino que es el propio proceso intercultural que debe incentivarlo, permitiendo que, siempre que sea necesario, se empoderen los grupos que necesiten afirmar más su identidad para que la igualdad pueda construirse y consolidarse en la propia interacción intercultural.

\subsection{RECONOCIMIENTO VS. REDISTRIBUCION}

En un conocido artículo, intitulado “¿De la redistribución al reconocimiento? Dilemas de la justicia en la era postsocialista”, Nancy Fraser (2001) discute de modo instigante y provocativo las relaciones entre igualdad y reconocimiento de las diferencias. Esta constituye la última tensión que queremos señalar en este trabajo. Vivimos en sociedades fuertemente marcadas por desigualdades socioeconómicas y por procesos de discriminación y prejuicio. Esta es una realidad que, en los países del continente, posee diversas configuraciones. Algunos se preguntan si esa preocupación por la interculturalidad no será una "trampa", en el sentido de que la misma nos aleja del verdadero problema del continente: la construcción de la justicia social. Por otro lado, también podríamos preguntarnos si es posible hoy en día desvincular las cuestiones relativas a la desigualdad de aquellas relativas a la diferencia. ¿No está, acaso, la pobreza "racializada", "etnizada" y "generificada", en el continente? La articulación entre redistribución y reconocimiento, desde la perspectiva de la interculturalidad crítica, es una exigencia de los tiempos actuales. Solamente una perspectiva de la interculturalidad que sea política, ética, educativa y epistemológica, podrá contribuir para que dicha articulación pueda consolidarse.

\section{CONSIDERACIONES FINALES}

El desarrollo de la educación intercultural en América Latina, con toda seguridad, se ha constituido en las últimas décadas en un proceso complejo, plural y original. La problemática, propia de un ámbito muy específico: la educación escolar indígena, se fue extendiendo hasta encontrarse, hoy, íntimamente ligada a los diferentes proyectos de estado y de sociedad que están en disputa en los diversos países del continente.

Consideramos que el desafío fundamental está en vincular las propuestas de educación intercultural a la perspectiva de la interculturalidad crítica. Se trata de una tarea compleja, ya que en la mayoría de los países en que se introdujo la interculturalidad en las políticas públicas, sobre todo en el ámbito educacional, en general predomina el enfoque funcional y el abordaje aditivo. Estos en muchos casos son folclorizantes y se limitan a incorporar en el currículo escolar componentes de las 
dos culturas de grupos sociales considerados "diferentes", particularmente indígenas $\mathrm{y}$ afrodescendientes.

Para que este tema pueda ser trabajado, es fundamental que integre el debate público en diferentes ámbitos sociales. En el caso de la educación, esta discusión aún está poco presente en las instituciones responsables por la formación de educadores, lo que constituye un gran obstáculo para su desarrollo. Sin embargo, las diferencias en las prácticas escolares y en las prácticas de la educación no formal están cobrando una sensibilidad y una visibilidad crecientes, a partir, muchas veces, de situaciones conflictivas. Esto permite afirmar que el debate sobre la educación intercultural está llamado a afianzarse en las sociedades latinoamericanas, incluso mediante la polémica y el enfrentamiento de posturas.

\section{BIBLIOGRAFIA}

Drelich, D. y Russo, K. (2009). Entre a Selva, a Costa e a Serra: a Educação Intercultural e o Reconhecimento das Diferenças no Peru. En: Candau, V. M. (org.), Educação Intercultural na América Latina: entre concepções, tensões e propostas. Río de Janeiro, 7 letras. (pp. 174197).

Fraser, N. (2001). Da redistribuição ao reconhecimento? Dilemas da justiça na era pós-socialista. En: Souza, J. (org.), Democracia Hoje: novos desafios para a teoria democrática contemporânea. Brasilia: Editora UnB (pp. 245-282).

López, L. E. y Sichra, I. (2004). La educación en áreas índigenas de América Latina: balances y perspectivas. En: Hernaiz, I. (org.), Educación en la diversidad. Experiencias y desafíos en la Educación Intercultural. Buenos Aires: IIPE (pp. 121-149).

Lopez-Hurtado Quiroz, L. E. (2007). Trece claves para entender la Interculturalidad en la Educación Latinoamericana. En: Prats, E. (coord.), Multiculturalismo y Educación para la Equidad. Barcelona: Octaedro-OEI (pp. 13-44).

Pedreira, S. y Sacavini, S. (2009). A Educação Intercultural na Bolívia: um caminho controverso. En: Candau, V. M. (org.), Educação Intercultural na América Latina: entre concepções, tensões e propostas. Río de Janeiro, 7 letras (pp. 198-227).

Tubino, F. (2005). La interculturalidad crítica como proyecto ético-político. Encuentro continental de educadores agustinos, Lima, enero 2005, http://oala.villanova.edu/congresos/educación/ lima-ponen-02.html 
Las colaboraciones, suscripciones y correspondencia deben ser dirigidas a la Dirección de la revista: Oficina de Publicaciones, Facultad de Filosofía y Humanidades, Universidad Austral de Chile, Casilla 567, Fono/Fax: 5663 221275, Valdivia, Chile. E-mail: eped@uach.cl

Las suscripciones incluyen los gastos de envío:

Chile: cheque o vale vista por \$10.000. Otros países: US\$ 15 .

El canje debe ser enviado a Biblioteca Central, Universidad Austral de Chile, Correo 2, Valdivia 
